\title{
Health care providers' knowledge, confidence, difficulties, and practices after completing a communication skills training program for advance care planning discussion in Japan
}

\author{
Hiroko Okada $^{1} \wedge$, Tatsuya Morita ${ }^{2}$, Takahiro Kiuchi ${ }^{1}$, Tsuyoshi Okuhara ${ }^{1}$, Yoshiyuki Kizawa ${ }^{3}$ \\ ${ }^{1}$ Department of Health Communication, School of Public Health, The University of Tokyo, Tokyo, Japan; ${ }^{2}$ Department of Palliative and Supportive \\ Care, Palliative Care Team, Seirei Mikatahara General Hospital, Hamamatsu, Shizuoka, Japan; ${ }^{3}$ Department of Palliative Medicine, Kobe University \\ Graduate School of Medicine, Kobe, Hyogo, Japan \\ Contributions: (I) Conception and design: H Okada, T Okuhara, T Kiuchi; (II) Administrative support: Y Kizawa, T Morita; (III) Provision of study \\ materials or patients: Y Kizawa; (IV) Collection and assembly of data: H Okada; (V) Data analysis and interpretation: All authors; (VI) Manuscript \\ writing: All authors; (VII) Final approval of manuscript: All authors. \\ Correspondence to: Hiroko Okada, RN, PhD. Department of Health Communication, School of Public Health, The University of Tokyo, 7-3-1 \\ Hongo, Bunkyo-ku, Tokyo 113-8655, Japan. Email: okadahiroko-tky@umin.ac.jp.
}

Background Advance care planning (ACP) is an important factor that affects the quality of end-oflife. However, health care providers are not able to sufficiently support patients' ACP due to their lack of knowledge and psychological burden. This study investigated how the knowledge, attitudes, and practices of health care providers who completed a communication skills training (CST) program for ACP discussion changed before and after the course.

Methods: Health care providers who participated in the Education for Implementing End of Life Discussion (E-FIELD) CST training program in Japan were surveyed at three time points: before, immediately after, and 6 months after training. The number of participants who completed all three surveys was 306. Knowledge, confidence, communication difficulties, number of discussions and completions of ACP were measured using a questionnaire.

Results: Participants' knowledge and confidence increased right after training compared with before training. The number of ACP discussions and completions increased significantly 6 months after training compared with before training and difficulties tended to decrease. Physicians maintained their confidence, which increased immediately after training until 6 months after training and their number of ACP discussions and completions also increased significantly 6 months after training compared with before training.

Conclusions: The CST program for ACP, E-FIELD, has the potential to increase the knowledge, confidence, and number of ACP discussions and completions used by physicians, nurses, and social workers, and also lower their difficulties.

Keywords: Advance care planning (ACP); end-of-life care; communication skills training (CST); health communication; physician-patient relations

Submitted Mar 19, 2021. Accepted for publication May 28, 2021.

doi: 10.21037/apm-21-642

View this article at: https://dx.doi.org/10.21037/apm-21-642

$\wedge$ ORCID: 0000-0001-7877-9753. 


\section{Introduction}

Advance care planning (ACP) is a decision-making process for end-of-life care that involves identifying goals and preferences for future treatment and care, discussing these goals and preferences with family and health care providers, and documenting and reviewing them as needed (1).

ACP has been shown to have many benefits for individuals, their families, and health care providers. The benefits to the individual include an increased sense of selfcontrol over their treatment, consideration and respect for their wishes about the treatment, place of care and endof-life care, and increased dialogue with family and health care providers (2-8). The benefits to the family include their reduced decision-making burden and the reduced stress, anxiety, and depression experienced after their family member's death (9). For health care providers, ACP has been reported to have the effect of reducing their moral distress and struggle with whether the medical care provided was the best for the patient (10). Despite these reported effects, most countries have reported a low percentage of health care providers who have completed the ACP (11-14).

In Japan, the Ministry of Health, Labor and Welfare has conducted a series of surveys on attitudes toward end-oflife care every 5 years since 1987. In 2007, it published the Guidelines for Decision-Making about End-of-Life Care (15). In addition, in 2018, these guidelines were revised to add the importance of ACP and the need to determine a surrogate decision-maker. In a 2016 survey of attitudes, however, although about $60 \%$ of Japanese reported having considered end-of-life care only $2.7 \%$ had discussed it in detail with their families or health care providers (16). In the same survey, less than $30 \%$ of physicians and nurses reported practicing ACP.

One of the most important and difficult issues in the end-of-life discussion is when to start talking about it (17). Many people have strong psychological barriers to talking about the end of life (18). Many Japanese people do not feel comfortable talking about the end of life and many want to spend the end of their lives without being aware of death $(19,20)$. Similarly, health care providers recognize the need and responsibility for discussing end-of-life issues with their patients, but are not comfortable with the subject or try to avoid it $(3,21-23)$. There is also a lack of knowledge about ACP among health care providers (24). As a result of delayed decision-making for these reasons, discussions are held only to decide whether or not to provide life-sustaining treatment when death is imminent (25). And the position of the family in Japan makes this issue even more difficult.
Both health care providers and patients recognize the family as a party, and health care providers need to communicate with both patients and families (26). Therefore, in order to promote ACP, it is important for healthcare providers to first develop communication skills to support patients' ACP and reduce the psychological barrier to initiating ACP discussions. Several training programs for health care providers have been developed and their effectiveness has been reported $(27,28)$. However, there are almost no reports of programs that focus specifically on communication skills training (CST) for healthcare providers on ACP. It has been reported that CST is effective in increasing the confidence of health care providers in difficult communication tasks, such as when telling bad news (29-31). These training may also contribute to the ability of health care providers to promote ACP.

Therefore, we developed Education for Implementing End of life Discussion (E-FIELD), a CST program to help health care providers face patients' ACP with confidence (32). This program also includes interprofessional CST to promote interprofessional work, which is one of the key elements in supporting patients' ACP. The E-FIELD program is the only training program of ACP discussion for healthcare providers in Japan and it is conducted on a nationwide scale.

The purpose of this study was to investigate how health care providers who attended the E-FIELD program changed before and after the course in terms of their ACP knowledge, confidence, communication difficulty, and practices.

We present the following article in accordance with the STROBE reporting checklist (available at https://dx.doi. org/10.21037/apm-21-642).

\section{Methods}

This was a longitudinal study of physicians, nurses, and social workers (SWs) who attended the E-FIELD before and after the intervention. The surveys were conducted between October 2017 and February 2018 in 12 locations across Japan where the E-FIELD took place.

\section{Intervention}

E-FIELD is a CST program that provides health care workers with competency to support their patients' ACP in hospital facilities. The program was developed by a working group consisting of four palliative care physicians, two nurse scientists, one SW, one ethicist, one legal scholar, 
Table 1 Details of E-FIELD

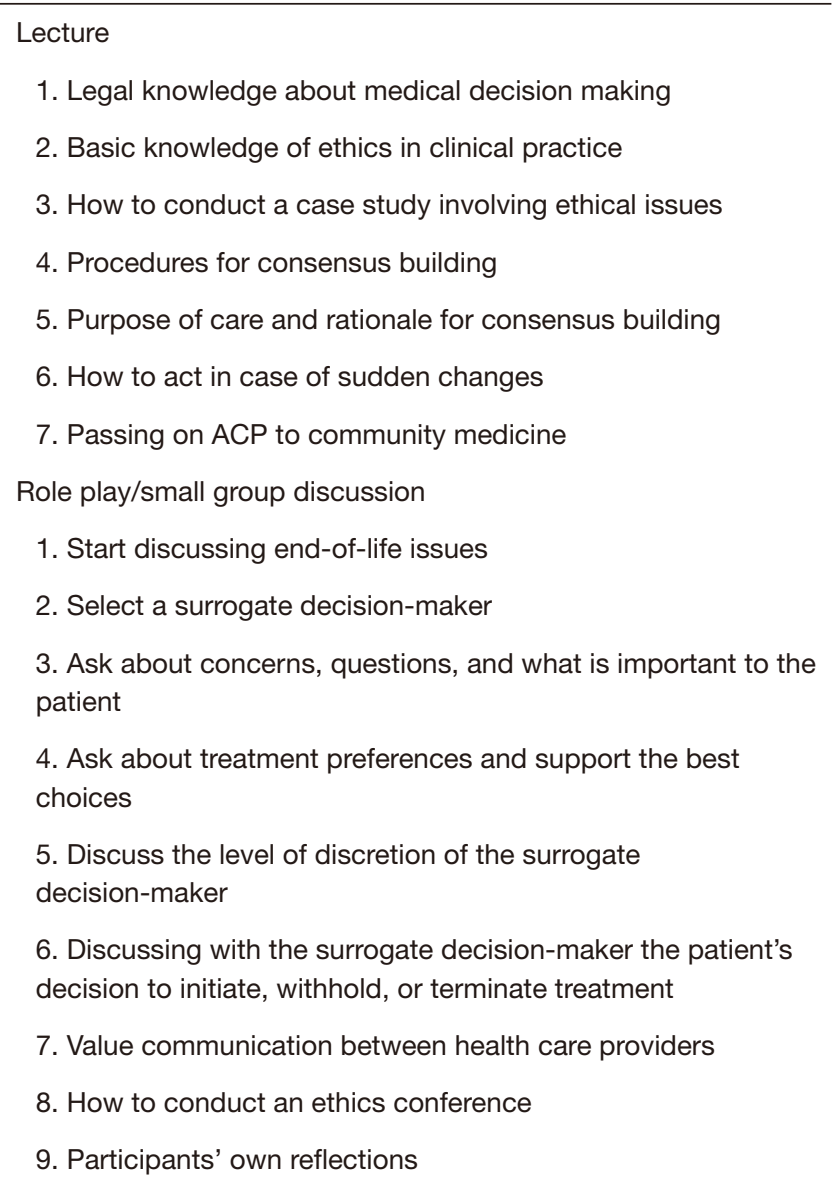

E-FIELD, Education for Implementing End of Life Discussion; ACP, advance care planning.

and one medical communication scholar. The contents of the program are shown in Table 1. These contents were selected through discussions within the working group based on a review of previous studies and the Guidelines for Decision-Making about End-of-Life Care developed by the Ministry of Health, Labour and Welfare (1-15,33). Some of the members of this group were also the developers of the guidelines.

The program was 2 days long with a participantcentered approach. And it was open to physicians, nurses, and SWs with at least three years of experience in treating, caring for, and counseling terminally ill patients. The program consisted of an ice-breaker, 4-hour textbased lecture using an original textbook common to all professions, 4 hours of small group discussions on a case requiring decision support, 4 hours of role-plays in ACPspecific scenarios, and discussions on these role-plays. The discussion covered typical topics such as decisions on start or interrupt life-sustaining treatment, as well as cases of family-driven decision making, which is a cultural issue in Japan. In addition, cases in which the best decisions differed depending on the profession were also discussed. Roleplays were conducted in scenarios specific to ACP, such as introduction to ACP, selection of a surrogate decisionmaker, and decision-making regarding life-sustaining treatment. In this program, the family was treated as a second party, considering the cultural characteristics of Japan. Therefore, in the role-plays, family members were placed in addition to the patient role. Discussions and role-plays were also conducted in settings that required adjustment of the patient-family relationship.

Discussions and role-playing were conducted in groups of four, each consisting of multiple professions, and one facilitator was assigned to each group. And they were conducted both in communication situations with patients and with other professions. Facilitators were palliative physicians, palliative care nurses, and medical ethicists with at least 3 years of clinical experience who participated in a 10-hour training workshop to facilitate communication skills.

\section{Data collection}

The inclusion criteria for this study were physicians, nurses, and SWs with at least three years of experience in treating, caring for, and counseling terminally ill patients who were participating in this training for the first time. Of the total number of participants in the training, all who met the criteria were included in the study. The surveys were conducted using self-administered questionnaires. The participants completed the questionnaires at the training site before and immediately after attending the program. They also completed a mailed questionnaire 6 months after attending the program. The survey took about 10 minutes to complete and no rewards were offered to participants. All participants were given an overview of the study by the researcher. If they wanted to participate, they then signed a consent form and completed the questionnaire. Six hundred and ninety-eight participants completed the survey before training, and 685 participants completed the survey immediately after training. Thirteen participants who missed a part of the training program or left early were excluded from the analysis. The training attrition rate was $1.8 \%$. After 6 months of training, 306 participants completed the survey. The follow-up rate was $44.7 \%$ (Figure 1). There was no significant difference in the proportion of 


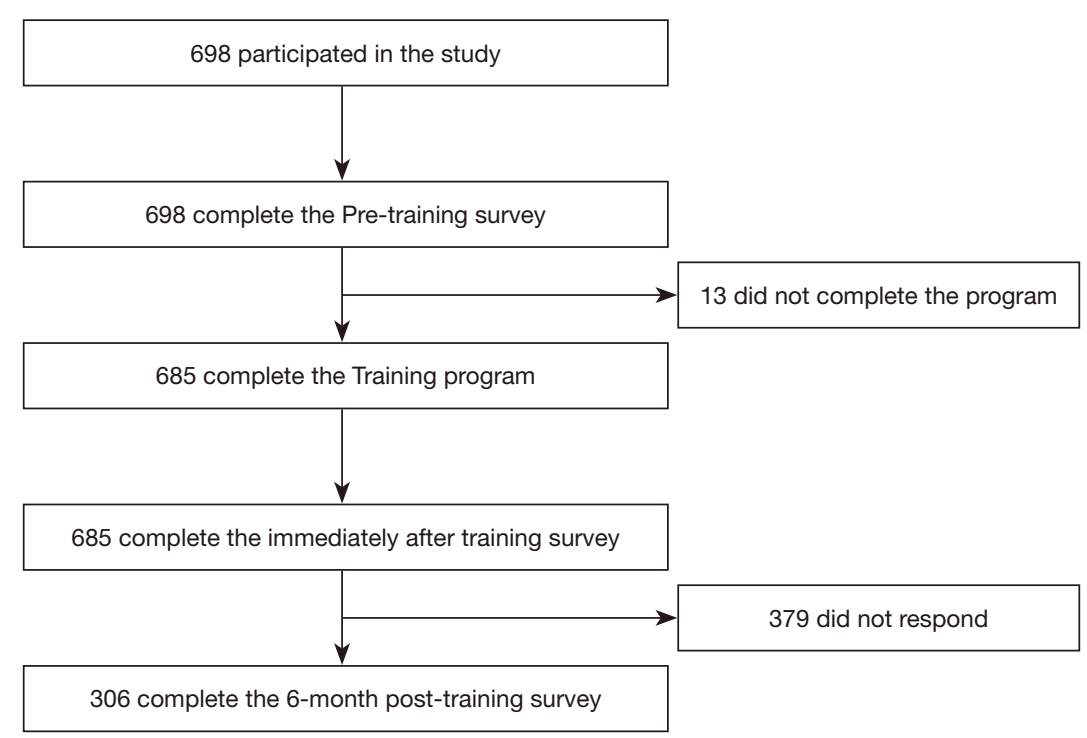

Figure 1 Study flow chart.

occupations of those who completed the 6-month training survey and those who left.

\section{Measurement}

\section{Sociodemographic characteristics}

The participants provided baseline responses for gender, age, workplace size, job title, years of clinical experience, and the number of patients in their charge who died in the past year.

\section{Knowledge}

We developed true/false questions about the knowledge necessary to support patients' ACP that is provided in the training program and checked their mastery of the knowledge. This questionnaire consisted of 23 questions, including four questions on guidelines for ACP, six questions on initiation and interruption of life-sustaining treatment, and 13 questions on the process of ACP. These questions were asked at three time points: before training, immediately after training, and 6 months after training.

\section{Confidence}

The participants' level of self-confidence in supporting ACP was examined using a 7 -point Likert scale (i.e., $0=$ not confident at all to $7=$ very confident).

Difficulty in communicating with terminally ill patients The communication module of the Nurses' Difficulty with
Cancer Care (NDCC) scale was used for the evaluation (34). The NDCC scale was developed based on a previous study that investigated nurses' difficulties in communicating with terminally ill patients $(35,36)$. The scale consists of 13 items, including "it is difficult to respond to patients immediately after they are given 'bad news' about their condition or prognosis" and "I cannot take enough time to talk with patients". Each question is rated using a 6-point Likert scale (i.e., $0=$ not at all agree, $1=$ agree, to $6=$ very much agree). This scale was surveyed at two time points: before training and 6 months after training.

\section{ACP discussion and completion}

The recommended ACP support was identified with reference to the results of the international Delphi survey by European Association for Palliative Care and the Quality Indicator development study on ACP (1,33). Specifically, 20 items were selected, including "explaining the advantages and disadvantages of end-of-life care that may be an option" and "encouraging patients to choose a surrogate decisionmaker". The implementation status of these items was evaluated using a 5 -point Likert scale (i.e., $0=$ never to $5=$ always). The number of respondents who answered "mostly" or "always" was calculated.

\section{Ethical considerations}

The study was conducted in accordance with the Declaration of Helsinki (as revised in 2013). All study 
Table 2 Participants characteristics

\begin{tabular}{|c|c|c|c|c|c|c|}
\hline Variables & \multicolumn{2}{|c|}{ Nurse $(n=160)$} & \multicolumn{2}{|c|}{ SW $(n=33)$} & \multicolumn{2}{|c|}{ Physician (n=113) } \\
\hline \multicolumn{7}{|l|}{ Sex } \\
\hline Male & 7 & 4.4 & 8 & 24.2 & 88 & 77.9 \\
\hline Female & 153 & 95.6 & 25 & 75.8 & 25 & 22.1 \\
\hline Years of clinical experience, mean $\pm S D$ & \multicolumn{2}{|c|}{$20.0 \pm 8.0$} & \multicolumn{2}{|c|}{$11.2 \pm 7.0$} & \multicolumn{2}{|c|}{$22.3 \pm 9.3$} \\
\hline $\begin{array}{l}\text { Number of patients in charge who died in the } \\
\text { past year, mean } \pm S D\end{array}$ & \multicolumn{2}{|c|}{$40.2 \pm 49.6$} & \multicolumn{2}{|c|}{$26.9 \pm 17.5$} & \multicolumn{2}{|c|}{$28.5 \pm 31.4$} \\
\hline \multicolumn{7}{|l|}{ Workplace } \\
\hline
\end{tabular}

SD, standard deviation; SW, social worker.

participants provided their written informed consent and the study design was approved by the University of Tokyo Medical School Ethics Committee and Institutional Review Board (No. 11270).

\section{Statistical analysis}

Data from those who completed all three questionnaire surveys were included in the analysis. To describe the participants' sociodemographic characteristics, we described frequencies and percentages for the categorical variables and means and standard deviations for the continuous variables. For knowledge and self-efficacy, we first described the scores at three time points: before training, immediately after training, and 6 months after training. Next, repeatedmeasures analyses of covariance with years of clinical experience and the number of patients in charge who died in 1 year as covariates were conducted at the three time points to examine the differences by occupation.

For difficulty in communicating with terminally ill patients and ACP support for patients, we first described the scores at two time points: before training and 6 months after training, respectively. Next, to examine the differences by job type, we conducted a repeated-measures analysis of covariance with years of clinical experience and the number of patients in charge who died in 1 year as covariates. For all scores, we also performed a repeated-measures analysis of covariance by job type, with years of clinical experience and the number of patients in charge who died in 1 year as covariates.

Data with missing values were removed, and only complete data were analyzed. All statistical tests were considered statistically significant with a $\mathrm{P}$ value $<0.05$. We used SPSS software (v. 26.0; IBM SPSS, Armonk, NY, USA) for statistical analysis.

\section{Results}

Gender, age, years of clinical experience, number of visits per year, and type of facility affiliation were significantly different among the job categories (Table 2). Gender and job type were significantly correlated, with nurses and SWs tending to be more often women and physicians more often men $(r=0.72, \mathrm{P}<0.001)$. Age and years of clinical experience were also correlated significantly, with older age tending to be associated with more years of clinical experience $(r=0.87$, $\mathrm{P}<0.001$ ).

Knowledge scores tended to increase from before training to immediately after training, both overall and in each occupational group (Table 3). In addition, there was a statistically significant increase for health care providers overall and for nurses and SWs. Six months after training, the knowledge was significantly higher for overall health care providers and SWs compared with before training. Overall, the knowledge tended to decrease immediately after training to 6 months after training. There was a 


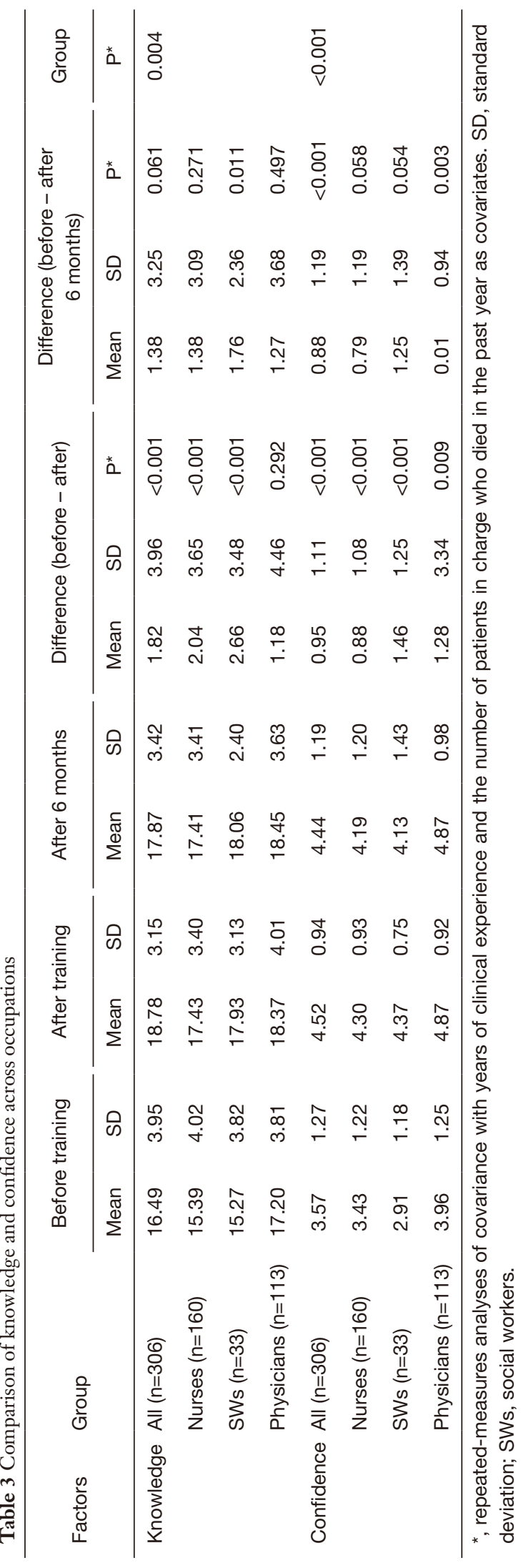

statistically significant difference in knowledge between occupational groups, especially between physicians and nurses $(\mathrm{P}=0.003)$.

In terms of the percentage of correct answers by item, the percentage of correct answers tended to be higher for most questions immediately after training and 6 months after training than before training. In particular, the percentage of correct answers to questions about the content of the guidelines increased significantly. However, the percentage of correct answers to the question on whether gastric banding and ventilators can be interrupted increased immediately after training compared with before training, but decreased to the same level or lower 6 months after training.

Confidence scores increased significantly from before training to immediately after training overall and in all occupational groups (Table 3). Six months after training, confidence was significantly higher in the overall and physician groups compared with before training.

Overall, there was a significant decrease in difficulty 6 months after training compared with before training (Table 4). There was no difference in difficulty among the occupational groups.

The number of ACP discussions and completions increased significantly from before training to 6 months after training for the health care providers overall and for physicians (Table 4). There was a difference in the number of ACP discussions and completions among the occupational groups, with SWs having significantly more ACP discussions and completions than physicians and nurses $(\mathrm{P}=0.046$ and 0.011 respectively).

\section{Discussion}

In this study, we conducted a CST program for health care providers to support their patients' ACP and evaluated the participants' knowledge, confidence, difficulty, and discussions and completions of ACP at three time points: before training, after training, and 6 months after training. As a result, all participants' knowledge and confidence increased immediately after training compared with before training. The number of ACP discussions and completions increased significantly 6 months after training compared with before training while their difficulties tended to decrease. SWs maintained their increased knowledge from the ACP training until 6 months later. Physicians maintained their confidence, which increased immediately after training until 6 months after training and their number of ACP discussions 
Table 4 Comparison of difficulties and practices across occupations

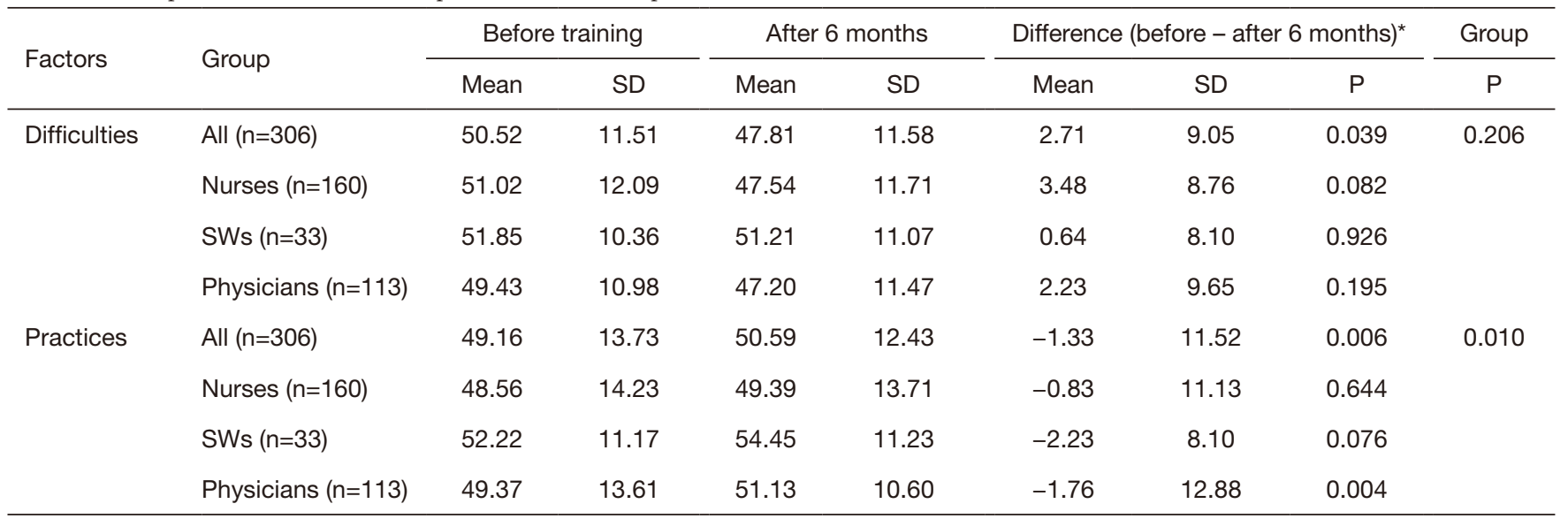

*, repeated-measures analyses of covariance with years of clinical experience and the number of patients in charge who died in the past year as covariates. SD, standard deviation; SWs, social workers.

and completions also increased significantly 6 months after training compared with before training.

The correct answer ratio for the knowledge test increased to about $75 \%$ after training. Especially for SWs, their increased score was significant and their knowledge was maintained for a long time. According to Knowles' adult learning theory, adults are motivated to learn to obtain answers and solve problems (37). The learning experience is most effective when education is delivered in a way that meets the learner's needs. Although all study participants were engaged in ACP-related work, SWs had performed more ACP support than did any other occupational group before training. SWs may have had many issues and questions about ACP and support methods through providing ACP support to many patients and felt the need to resolve them more strongly. In addition, knowledge gained through demonstrations and discussions is more likely to be retained in memory than knowledge gained by watching or listening to a lecture (38). Furthermore, knowledge gained through practical experience is much more likely to be retained (38). This training included a lot of small group discussions and role playing. The SWs experienced more ACP discussions and completions after training, which may have led to their retention of knowledge.

The percentage of correct answers to questions about the guidelines increased significantly after training compared with before training in all occupations. On the other hand, the percentage of correct answers to questions about situations in which it is difficult to make decisions, such as handling the wishes of family members when the patient lacks decision-making capacity or interrupting life-sustaining treatment, remained low after training. These items also required a change in perception of the assumptions that are conventionally adopted in clinical practice, such as that ventilators should be started on the assumption that they will not be interrupted. According to Mezirow's transformative learning theory, learning is the process of creating new or modified interpretations of the meaning of one's experiences (39). Rational discussion (discourse) with others is essential for this process. Learning that involves greater cognitive transformation requires a process of reintegrating new perceptions into one's life by repeating the process of transforming one's perceptions of assumptions through the acquisition of knowledge and skills, building competence and confidence through experience, and then discussing them with others. Therefore, the participants will be able to further raise their stage of transformative learning if they have the opportunity to continuously update their knowledge and engage in discussions (40).

Confidence was higher among physicians before training than in other occupational groups and was also significantly higher after training. A survey of nurses has shown that higher knowledge of ACP and higher confidence are associated with the number of ACP discussions and completions and comprehensive end-of-life care (41). The knowledge of physicians in this study was higher than other occupational groups both before and after training, and their knowledge immediately after training was significantly correlated with confidence 6 months after training $(r=0.32, \mathrm{P}<0.001)$. Similarly, confidence immediately after 


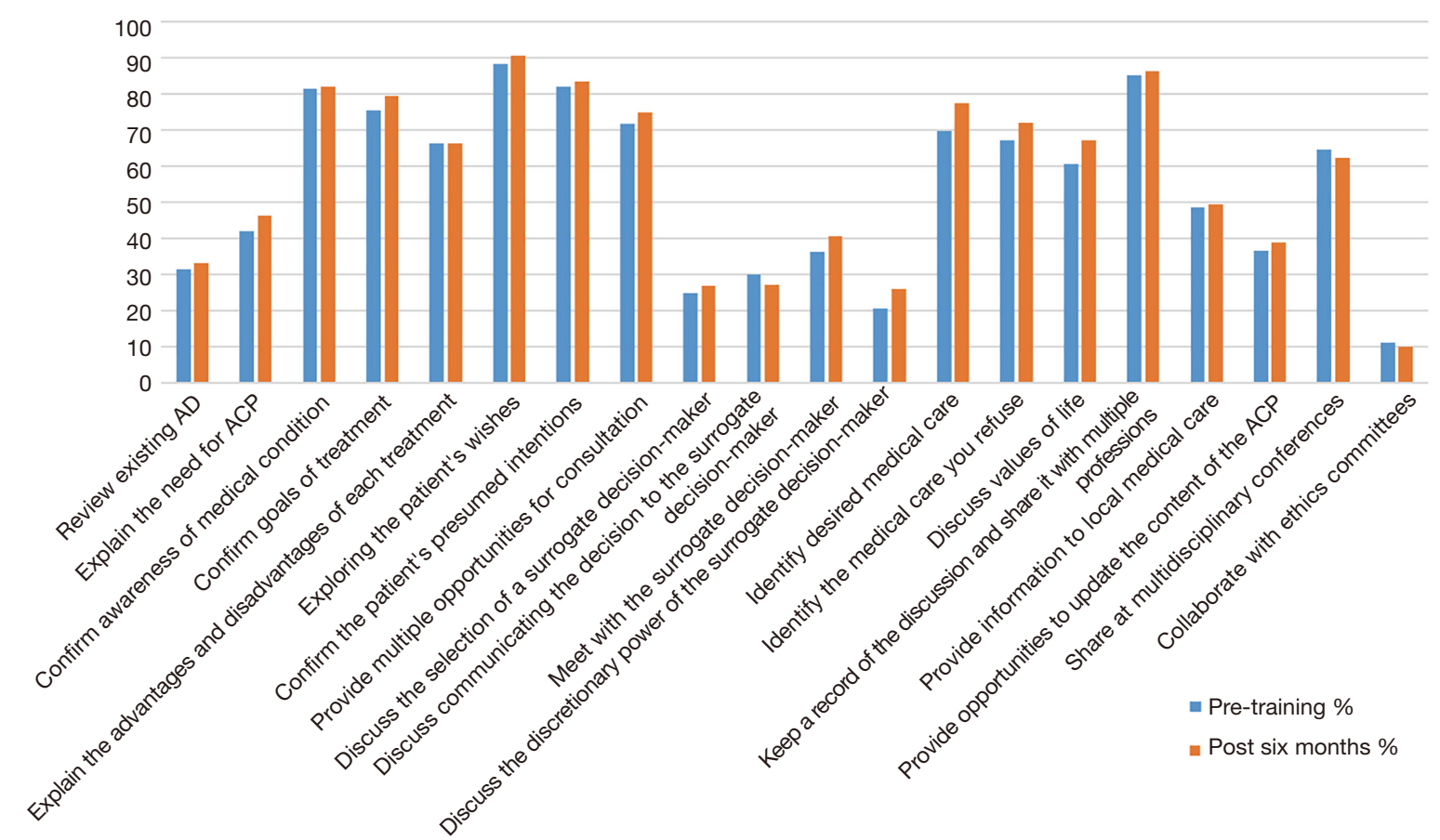

Figure 2 Changes in the percentage of ACP practices for each item before and after training $(\mathrm{N}=306)$. ACP, advance care planning.

training was significantly correlated with the number of ACP discussions and completions 6 months after training $(\mathrm{r}=0.26, \mathrm{P}<0.001)$. This suggests that even in physicians' ACP support, gaining knowledge leads to confidence, and gaining confidence leads to better practice.

Health care providers feel psychologically burdened to talk with their patients about end-of-life care, which is one of the reasons why end-of-life discussions often are inadequate or do not take place with sufficient content or time (3,21-23). Also, being knowledgeable and confident about end-of-life care is associated with being comfortable communicating with patients and families at the end of life (42). Although the study participants were health care providers engaged in palliative care, their confidence in ACP support before training was low and more than half of them felt difficulties when their patients talked about death. In our study, despite an increase in the number of ACP discussions and completions, participants' difficulties in communicating with patients at the end of life tended to be lower overall, although this result did not reach significance. This may be because the participants repeatedly practiced demonstrations and role played various difficult communication scenarios during the training program, which increased their confidence in communication and lowered their difficulties.
The selection of a surrogate decision maker, sharing the decision making, and determining the level of discretion should be included in the ACP (1). Therefore, these course contents were also focused on in the ACP facilitator training program. However, less than half of the participants implemented the support related to surrogate decision makers both before and after training (Figure 2). In Asian countries, family members often make medical decisions on behalf of the patient regardless of the patient's own decision-making capacity (26). In many cases, patients also want to entrust decisions to their families $(1,43)$. Therefore, both health care providers and patients may feel uncomfortable or that it is unnecessary to dare to appoint a family member as a "surrogate decision maker", sign the legal documents, or decide the degree of discretion. However, it has been reported that outside of Japan, the percentage of elderly people who have selected a surrogate decision maker was more than $60 \%$. Thus, it would be difficult to apply foreign examples of support related to surrogate decision makers (44). This is an area that should be reexamined based on data from Japanese health care providers and patients to find a method of support that is culturally appropriate for Japanese people.

This study needs to be interpreted under several limitations. The study was conducted using a self-report questionnaire, 
which may have introduced bias. The lack of a control group and the lack of randomized assignment may have led to confounding in areas other than the data obtained. The number of SW participants was small and meaningful differences may not have been detected. The participants were health care providers engaged in palliative care, which limits the extent to which the results can be generalized.

\section{Conclusions}

The CST program for ACP discussion, E-FIELD, has the potential to increase the knowledge, confidence, and number of discussions and completions of physicians, nurses, and SWs in supporting ACP for their patients, and reduce their difficulties. Physicians and nurses' knowledge and confidence tend to decline within 6 months after training; thus, continuous education is required. Especially for support of difficult decisions such as interruption of lifesustaining treatment, it may be necessary to periodically update knowledge, brush up communication skills, and provide opportunities for discussions. Support methods for appointing a proxy decision maker must be developed to fit Japanese culture.

\section{Acknowledgments}

We thank Peter Fogarty, MA English 1st Class, from Edanz Group (https://en-author-services.edanzgroup.com/), for editing a draft of this manuscript.

Funding: This study was funded by the National Project for Improving End-of-Life Care 2017.

\section{Footnote}

Reporting Checklist: The authors have completed the STROBE reporting checklist. Available at https://dx.doi. org/10.21037/apm-21-642

Data Sharing Statement: Available at https://dx.doi. org/10.21037/apm-21-642

Peer Review File: Available at https://dx.doi.org/10.21037/ apm-21-642

Conflicts of Interest: All authors have completed the ICMJE uniform disclosure form (available at https://dx.doi. org/10.21037/apm-21-642). The authors have no conflicts of interest to declare.
Ethical Statement: The authors are accountable for all aspects of the work in ensuring that questions related to the accuracy or integrity of any part of the work are appropriately investigated and resolved. The study was conducted in accordance with the Declaration of Helsinki (as revised in 2013). All study participants provided their written informed consent and the study design was approved by the University of Tokyo Medical School Ethics Committee and Institutional Review Board (No. 11270).

Open Access Statement: This is an Open Access article distributed in accordance with the Creative Commons Attribution-NonCommercial-NoDerivs 4.0 International License (CC BY-NC-ND 4.0), which permits the noncommercial replication and distribution of the article with the strict proviso that no changes or edits are made and the original work is properly cited (including links to both the formal publication through the relevant DOI and the license). See: https://creativecommons.org/licenses/by-nc-nd/4.0/.

\section{References}

1. Rietjens JAC, Sudore RL, Connolly M, et al. European Association for Palliative Care: Definition and recommendations for advance care planning: An international consensus supported by the European Association for Palliative Care. Lancet Oncol 2017;18:e543-51.

2. Morrison RS, Chichin E, Carter J, et al. The effect of a social work intervention to enhance advance care planning documentation in the nursing home. J Am Geriatr Soc 2005;53:290-4.

3. Murray L, Butow PN. Advance care planning in motor neuron disease: A systematic review. Palliat Support Care 2016;14:411-32.

4. Martin RS, Hayes B, Gregorevic K, et al. The effects of advance care planning interventions on nursing home residents: a systematic review. J Am Med Dir Assoc 2016;17:284-93.

5. Ke LS, Huang X, O'Connor M, et al. Nurses' views regarding implementing advance care planning for older people: a systematic review and synthesis of qualitative studies. J Clin Nurs 2015;24:2057-73.

6. Meeker MA, Jezewski MA. Family decision making at end of life. Palliat Support Care 2005;3:131-42.

7. Song K, Amatya B, Voutier C et al. Advance care planning in patients with primary malignant brain tumors: A systematic review. Front Oncol 2016;6:223. 
8. Hickman SE, Keevern E, Hammes B. Use of the Physician Orders for Life-Sustaining Treatment program in the clinical setting: A systematic review of the literature. J Am Geriatr Soc 2015;63:341-50.

9. Detering KM, Hancock AD, Reade MC, et al. The impact of advance care planning on end of life care in elderly patients: Randomised controlled trial. BMJ 2010;340:c1345.

10. Elpern EH, Covert B, Kleinpell R. Moral distress of staff nurses in a medical intensive care unit. Am J Crit Care 2005;14:523-30.

11. Aw D, Hayhoe B, Smajdor A, et al. Advance care planning and the older patient. QJM 2012;105:225-30.

12. Evans N, Bausewein C, Menaca A, et al. A critical review of advance directives in Germany: Attitudes, use and healthcare professionals' compliance. Patient Educ Couns 2012;87:277-88.

13. Simon-Lorda P, Tamayo-Velázquez MI, Barrio-Cantalejo IM. Advance directives in Spain. Perspectives from a medical bioethicist approach. Bioethics 2008;22:346-54.

14. van Wijmen MP, Rurup ML, Pasman HR, et al. Advance directives in the Netherlands: An empirical contribution to the exploration of a cross-cultural perspective on advance directives. Bioethics 2010;24:118-26.

15. Japanese Ministry of Health, Labour and Welfare: Guidelines for Decision-Making about End-ofLife Care. 2018. Available online: https://www.mhlw. go.jp/file/04-Houdouhappyou-10802000-IseikyokuShidouka/0000197701.pdf (last accessed January 20, 2021).

16. Japanese Ministry of Health, Labour and Welfare: Report of a Survey of Perceptions Regarding End of Life Care. 2013. Available online: https://www.mhlw.go.jp/file/05Shingikai-10801000-Iseikyoku-Soumuka/0000041847_3. pdf (last accessed August 1, 2019).

17. Sharp T, Moran E, Kuhn I, et al. Do the elderly have a voice? Advance care planning discussions with frail and older individuals: A systematic literature review and narrative synthesis. Br J Gen Pract 2013;63:e657-68.

18. Nilchaikovit T, Hill JM, Holland JC. The effects of culture on illness behavior and medical care. Asian and American differences. Gen Hosp Psychiatry 1993;15:41-50.

19. Akechi T, Akazawa T, Komori Y, et al. Dignity therapy: Preliminary cross-cultural findings regarding implementation among Japanese advanced cancer patients. Palliat Med 2012;26:768-9.

20. Miyashita M, Sanjo M, Morita T, et al. Good death in cancer care: A nationwide quantitative study. Ann Oncol 2007;18:1090-7.
21. Koh SJ, Kim S, Kim J. Communication for end-oflife care planning among Korean patients with terminal cancer: A context-oriented model. Palliat Support Care 2016;14:69-76.

22. Miovic M, Block S. Psychiatric disorders in advanced cancer. Cancer 2007;110:1665-76.

23. Layson RT, Adelman HM, Wallach PM, et al. Discussions about the use of life-sustaining treatments: A literature review of physicians' and patients' attitudes and practices. End of Life Study Group. J Clin Ethics 1994;5:195-203.

24. Kermel-Schiffman I, Werner P. Knowledge regarding advance care planning: A systematic review. Arch Gerontol Geriatr 2017;73:133-42.

25. Johnson S, Butow P, Kerridge I, et al. Advance care planning for cancer patients: A systematic review of perceptions and experiences of patients, families, and healthcare providers. Psychooncology 2016;25:362-86.

26. Cheng SY, Lin CP, Chan HY, et al. Advance care planning in Asian culture. Jpn J Clin Oncol 2020;50:976-89.

27. MacKenzie MA, Smith-Howell E, Bomba PA, et al. Respecting Choices and Related Models of Advance Care Planning: A Systematic Review of Published Evidence. Am J Hosp Palliat Care 2018;35:897-907.

28. Pecanac KE, Repenshek MF, Tennenbaum D, et al. Respecting Choices $®$ and advance directives in a diverse community. J Palliat Med 2014;17:282-7.

29. Moore PM, Rivera S, Bravo-Soto GA, et al. Communication skills training for healthcare professionals working with people who have cancer. Cochrane Database Syst Rev 2018;7:CD003751.

30. Walczak A, Butow PN, Bu S, et al. A systematic review of evidence for end-of-life communication interventions: Who do they target, how are they structured and do they work? Patient Educ Couns 2016;99:3-16.

31. LeBlanc TW, Temel JS, Helft PR. "How Much Time Do I Have?": Communicating Prognosis in the Era of Exceptional Responders. Am Soc Clin Oncol Educ Book 2018;38:787-94.

32. Miura H, Kizawa Y, Bito S, et al. Benefits of the Japanese version of the advance care planning facilitators' education program. Geriatr Gerontol Int 2017;17:350-2.

33. Sinuff T, Dodek P, You JJ, et al. Improving end-of-life communication and decision making: The development of a conceptual framework and quality indicators. J Pain Symptom Manage 2015;49:1070-80.

34. Onodera M, Kumata M, Ogiri N, et al. Development of scale to measure nurses' difficulty with cancer care (NDCC). Palliative Care Research 2013;8:240-7. 
35. Sasahara T, Miyashita M, Kawa M, et al. Difficulties encountered by nurses in the care of terminally ill cancer patients in general hospitals in Japan. Palliat Med 2003; 17:520-6.

36. Sasahara T, Miyashita M, Kawa M, et al. Factors associated with difficulties encountered by nurses in the care of terminally ill cancer patients in hospitals in Japan. Palliat Support Care 2005;3:15-22.

37. Knowles MS. The Modern Practice of Adult Education: From Pedagogy to Andragogy. 2nd edition. New York, NY: The Adult Education Company, 1980.

38. Dale E. Audio-visual Methods in Teaching. 3rd edition. New York, NY: Dryden Press, Holt, Rinehart \& Winston, 1969.

39. Mezirow J. Transformative Dimensions of Adult Learning. San Francisco, CA: Jossey Bass Higher \& Adult Education, 1991.

40. Yeh DD, Park YS. Improving learning efficiency of

Cite this article as: Okada $\mathrm{H}$, Morita T, Kiuchi T, Okuhara T, Kizawa Y. Health care providers' knowledge, confidence, difficulties, and practices after completing a communication skills training program for advance care planning discussion in Japan. Ann Palliat Med 2021;10(7):7225-7235. doi: 10.21037/apm21-642 factual knowledge in medical education. J Surg Educ 2015;72:882-9.

41. Ludwick R, Baughman KR, Jarjoura D, et al. CE: Original research: Advance care planning: An exploration of the beliefs, self-efficacy, education, and practices of RNs and LPNs. Am J Nurs 2018;118:26-32.

42. Coffey A, McCarthy G, Weathers E, et al. Nurses' knowledge of advance directives and perceived confidence in end-of-life care: A cross-sectional study in five countries. Int J Nurs Pract 2016;22:247-57.

43. Lee MC, Hinderer KA, Kehl KA. A systematic review of advance directives and advance care planning in Chinese people from eastern and western culture. J Hosp Palliat Nurse 2014;16:76-85.

44. Howard M, Bernard C, Klein D, et al. Older patient engagement in advance care planning in Canadian primary care practices: Results of a multisite survey. Can Fam Physician 2018;64:371-7. 